\title{
Stress Model of Axial Flow Threshing \& Separating Device and Trajectory Simulation of Rice Grains
}

\author{
Liu Ying-Nan and Yi Shu-Juan \\ Institute of Information Technology, Heilongjiang Bayi Agricultural University, Daqing \\ 163319, China \\ Dqpilyn@126.com
}

\begin{abstract}
To reveal stress distribution rules of grain separating process in assembled axial flow. threshing and separating device, regarding assembled axial flow threshing and separating device as object, High speed imaging technology is adopted in the shooting technique, stress model was set based on the stress principle, and stress distribution rules was obtained by simulation technology. Grain, broken ear movement and the movement as a whole and free movement of the grain threshing process is through the analysis of the EXCEL drawing, The test results show that distribution rules is irregular in grain separating process, is that, stress fluctuates at axial direction, and two peak values appear at early and middle threshing stage respectively; threshing and separating process finishes about 2.35 meters from the feeding mouth ataxial direction. Off the spike in threshing and separating space motion anstable trajectory is a parabola, the speed decreased with the passage of time. No butside interference in the most free grain for approximate straight movement, in the Gredium of molion state, and axis direction and a certain angle.Therefore, grain rotors distribute at axial direction and rest results are consistent with theory analysis.
\end{abstract}

Keywords: grain; axialflow threshing and separating device; stress; test

\section{Introduction}

Grain deformates under external force such as bar tooth after grain is fed in threshing cylinder. Additional intern force, a interaction force was generated because relative position of various parts inside grain changes under external force. Additional internal force is affected by exterhal force and changes correspondingly with the increase and decrease of external orce. When the additional internal force affected by external force exceeds the inherent strength limit between grain and rice ear, grains began to fall. So it is a direct relationship between additional internal force and damage degree in grain threshing process. Introducing the stress concept is to express the distribution of additional internal force affected by external force in the cross section. According to the stres principle, we can know that grain will be damaged when the stress exceeds the grain strength and stress is direct factor to damage components. Therefore, it's important to set the stress model in the grain threshing and separating process. Stress distribution can be analyzed in threshing and separating process and threshing and separating nature can be revealed based on the stress model, which helps to study distribution rules of grain rotors in theory. The stress model was set by stress principle, and the stress distribution of the grain threshing and separating process was analyzed base stress model, and verified by experiments to provide theoretical foundation for the best effect, the proper components and optimization of threshing and separating process in this paper.On Grain overall motion process, broken panicle threshing process and different drum speed free grain movement in the process of analysis and research. It provides some reference value for further improving the performance of the drum. 


\section{Determination of Grain Stress Model}

The stress on each part is different, and the stress on force point is affected by the direction. Through the analysis we can know that the stress in per unit area can be divided into two directions, is that, positive stress and shear stress. The direction which shear stress is zero is named as the main direction, plane which is vertical to the main direction is named as the main plane, and the positive stress in the main plane is called the main stress, represented by $\sigma \mathrm{p}$.

Collecting any infinitesimal tetrahedron from force body to analyze the stress and determine the main stress, Figure 1 shows that the three surfaces of collected tetrahedron are parallel to the coordinate surface. The edges parallel to the coordinate axis are represented by Dy, DZ, DX, the cosine between the normal on another surface of tetrahedron and the axes by $\mathrm{L}, \mathrm{m}, \mathrm{n}$, and the balance equations of each force on $\mathrm{x}$ axis are shown as:

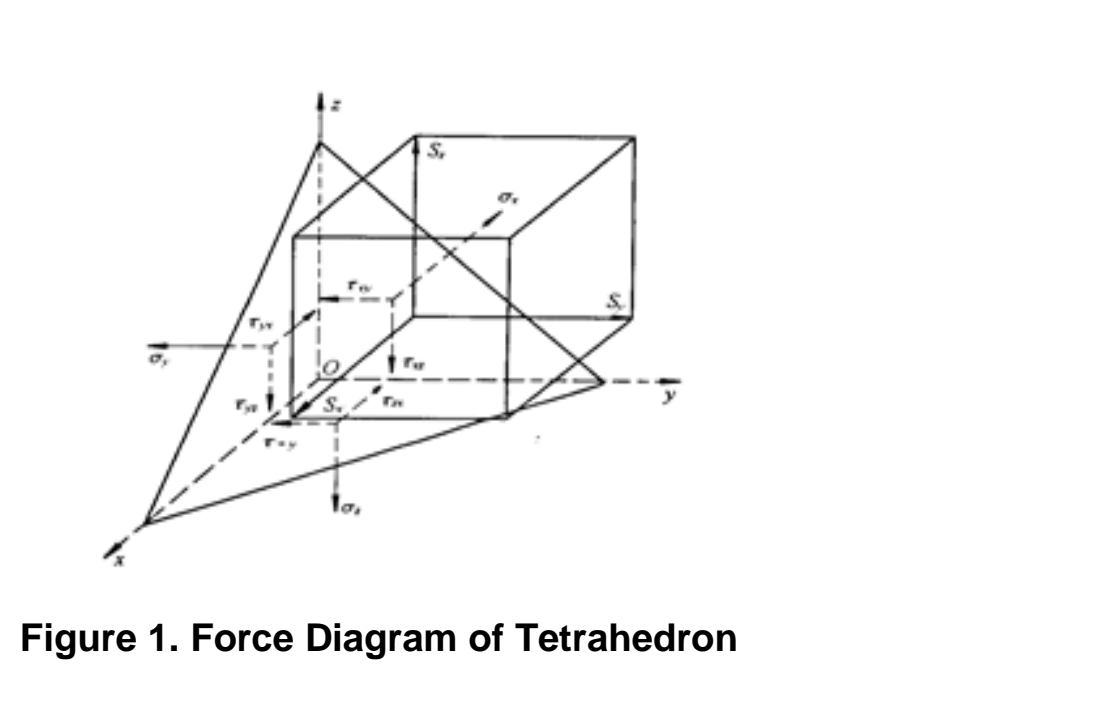

Conclusion by the geometric relationships between a plane figure projections:

$\frac{1}{2} \mathbf{d} \mathbf{y d x}=\mathbf{m a} \mathbf{A}, \frac{1}{2} \mathrm{~d} z d x=m d A, \frac{1}{2} d x d y=n d A$

Arrange:

$$
\begin{aligned}
& -\sigma_{x} \frac{1}{2} d y d z-\tau_{y z} \frac{1}{2} d x d z-\tau_{z x} \frac{1}{2} d x d y+S_{x} d A=0 \\
& S_{x}=\varnothing \sigma_{x}+m \tau_{y x}+n \tau_{z x}
\end{aligned}
$$

Similar to the $y, z$ axis projection:

$$
\begin{aligned}
S_{y} & =l \tau_{x y}+m \sigma_{y}+n \tau_{z y} \\
S_{z} & =l \tau_{x z}+m \tau_{y z}+n \sigma_{z}
\end{aligned}
$$

Where Sx, Sy, Sz are projection of the whole stress on rake surface respectively in the y, zaxis.

According to the concept of maximum stress:

$$
S_{x}=l \sigma_{p} 、 S_{y}=m \sigma_{p} 、 S_{z}=n \sigma_{p}
$$

By the geometry relations:

$l^{2}+m^{2}+n^{2}=1$

There are:

$$
\sigma_{p}^{3}-I_{1} \sigma_{p}^{2}+I_{2} \sigma_{p}-I_{3}=0
$$

Where I1: tensor invariant varible in first stress 
$I_{1}=\sigma_{x}+\sigma_{y}+\sigma_{z}$

I2: varible in second stress

$I_{2}=\sigma_{y} \sigma_{z}+\sigma_{x} \sigma_{z}+\sigma_{x} \sigma_{y}-\tau_{y z}^{2}-\tau_{x z}^{2}-\tau_{x y}^{2}$

I3: varible in the third stress

$$
I_{3}=\left|\begin{array}{lll}
\sigma_{x} & \tau_{y z} & \tau_{z x} \\
\tau_{x y} & \sigma_{y} & \tau_{z y} \\
\tau_{x z} & \tau_{y z} & \sigma_{z}
\end{array}\right|
$$

Where, $\sigma_{x} 、 \tau_{x y}, \tau_{x z}, \sigma_{z} 、 \tau_{z x} 、 \tau_{z y} 、 \sigma_{y} 、 \tau_{y x} 、 \tau_{y z}$ can be obtained through analyzing deformation figure according to the principle of stress:

$$
\begin{aligned}
& \sigma_{y}=\frac{F}{A}=\frac{F_{n}}{d s * a} \\
& \tau_{y z}=\frac{T}{\alpha h b^{2}}=\frac{\left(F_{g} f_{s}-\mathrm{F}_{\mathrm{n}} f_{s}\right) h}{\alpha^{*} a^{*} d s} \sin \beta+\frac{F_{d} f_{s} a}{\alpha h^{*} d s} \cos \beta \\
& \tau_{y x}=\frac{T}{\alpha h b^{2}}=\frac{\left(F_{g} f_{s}-F_{n} f_{s}\right) h}{\alpha^{*} a * d s} \cos \beta+\frac{F_{d} f_{s} a}{\alpha h * d s} \sin \beta \\
& \sigma_{x}=\frac{F}{A}+\frac{M y}{I_{2}}=\frac{F_{d}}{d s^{*} h} \sin \beta+\frac{6 F+a}{h a^{4}} \cos \beta \\
& \tau_{x y}=\frac{T}{\alpha h b^{2}}=\frac{F_{d} f a}{\alpha h_{d s} d s} \beta \\
& \tau_{x z}=\frac{T}{\alpha h b^{2}}=\frac{F_{d} f_{s} g}{\alpha h \cos \beta} \\
& \sigma_{z}=\frac{F}{A} \frac{M y}{I_{z}}=\frac{F_{d}}{d s^{*} h} \cos \beta+\frac{6 F_{d} f_{s} a}{h a^{4}} \sin \beta \\
& \tau_{z x}=\frac{T}{\alpha h b^{2}}=\frac{F_{d} f_{s} a}{a h^{*} d s} \cos \beta
\end{aligned}
$$

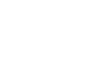

$$
\begin{aligned}
& +1
\end{aligned}
$$

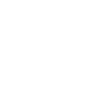

$$
\begin{aligned}
& \text { ( }
\end{aligned}
$$

$0_{x} 、 \tau_{x y} 、 \tau_{x z}$ : positive stress and shear stress on parallel surface of the normal and $\mathrm{x}$ axis;

$\sigma_{y} 、 \tau_{y x}, \tau_{y z}:$ positive stress and shear stress on parallel surface of the normal and $\mathrm{y}$ axis;

$\sigma_{z} 、 \tau_{z x}, \tau_{z y}$ : positive stress and shear stress on parallel surface of the normal and $\mathrm{z}$ axis;

Fn, Fg, Fd: are respectively upward force of cylinder surface and extrusion force of cover plate on the infinitesimal body; 
ds: the length of the infinitesimal section, ds $=10 \mathrm{~mm}$;

a: spiral blade distance, $\mathrm{a}=250 \mathrm{~mm}$;

$\mathrm{h}$ : infinitesimal section height, $\mathrm{h}=100 \mathrm{~mm}$;

fs: friction coefficient between grains and drum, $\mathrm{fs}=0.25$;

$\beta$ : angle of spiral vane $\beta=9$.;

$\alpha:$ is a coefficient related to the ratio of $\mathrm{h} / \mathrm{ds}, 0.2$.

Formula (6) - (14) into the formula (5), equations answer can be obtained, is that, the three main stresses $\sigma_{1} 、 \sigma_{2} 、 \sigma_{3}$ can be expressed as $\sigma_{3}<\sigma_{2}<\sigma_{1}$. Thus the main reason of affecting grain threshing is the main stress.

\section{Stress Test Results and Analysis}

\subsection{Simulation Analysis}

\section{(1) The Initial Conditions}

Under initial conditions: concave clearancea $30 \mathrm{~mm}$, and cylinder speed $600 \mathrm{r} / \mathrm{min}$, zenith distance $30 \mathrm{~mm}$, grain moisture content $15 \%$, and feeding amount $2 \mathrm{~kg} / \mathrm{s}$, based on Matlab, the formula (5) was solved by using ode45 command.

Critical condition: diameter seperated from cylinder $460 \mathrm{~mm}$, cylinder length: 2300 $\mathrm{mm}$, blade height $70 \mathrm{~mm}$, and spiral vane angle 10 degrees.

Set the initial conditions as: $\mathrm{t}=0, \sigma(0)=0$.

Test conditions are that the roller speed is $600 \mathrm{r} / \mathrm{min}$, feed rate is $0.5 \mathrm{~kg} / \mathrm{s}$, plate gear spacing is $16 \mathrm{~cm}$, guide angle of guide plate is 45 degrees, topdistance is $30 \mathrm{~mm}$, concave plate clearance is $30 \mathrm{~mm}$, concave grid size is $16 \mathrm{~mm} * 50 \mathrm{~mm}$, concave plate bag angle is 180 degrees. Test material is Kongyu 131 which was measured for that the average stem length is $68.2 \mathrm{~cm}$, and the moisture content was $21.5 \%$. The proportion for the grain and grass is 1.5:2.1; water content rate of kernel is $18.7 \%$.

The choice of online shooting is the high-speed camera which was made in U.S. with the shooting frequency of 500 frames /s and resolution of $1024 * 1024$.

\section{2. Analyzing the Results}

In Figure 2, we can see that stress in the axis direction tends to fluctuate in grain threshing and separating process, and its change is uneven, which the first peak value of main stress appears at the distance of 0.6 meters from grain feeding end, where rotors are off from grain to indicate that grains in the peak area are forced by the large stress. After the first peak, peak value appears once again at the distance of about 1.6 meters from the feeding end, then the main stress value declines. At the distance of about 2.3 meters from the feeding end, stress value is close to zero, which indicates that grain threshing and separating process finishes. 


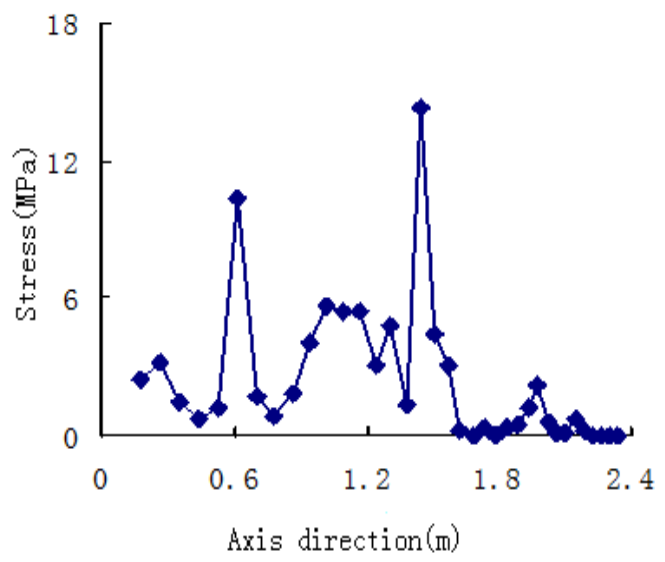

Figure 2. The Axial Distribution of Stress

\section{Analysis on the Whole Movement Process of Rice}

Under the condition of the out drum speed of is $500 \mathrm{r} / \mathrm{mnn}$, feeding quantity for the rice is $0.5 \mathrm{~kg} / \mathrm{s}$, plate tooth spacing is $16 \mathrm{~cm}$, the rate of moisture content in the grain is $21.5 \%$, the video image was slow which was collected by the high-speed camera, after the valley stream entering in axial flow off space, with the joint action for the push of guide plate and being grabbed power by board teeth, orming a certain thickness of rice layer, rice layer spiral surging forward along the roller axis direction, during the forward movement of rice layer, a part of the grain is out in force against tooth threshing board. A part of the grain threshing process is carried aut ander the action of the rub in the side tooth, the top threshing board tooth and the concave cambered surface. Due to repeated process of threshing, more than 99\% grain ultimately achieves the separation between the stem access into the furnaces. At the same time, it also produced a large number of short stem, leaf and other impurities and a small amount of off spike in the whole process of threshing.

\subsection{Analysis of the Movement Process of Broken Spike}

As shown in Figure 3, the-1oller speed is $500 \mathrm{r} / \mathrm{min}$, feed quantity is $0.5 \mathrm{~kg} / \mathrm{s}$, a group of pictures of the off spike threshing process in board tooth axial threshing off space were photographed by using high-speed video camera, the video image was analyzed which was captured by high-speed camera, threshing process threshing process of the off spike is divided into: the front, among and afterward of the threshing. The slow image can be observed that, the trajectory in the off drum is a parabola before breaking threshing. It is inverted after the off spike was hit by plate teeth at high speed for the first time; No. 1 grair Oyercomes the tension of the branch under the action of an external force, and reatizes the separation between the stem and branch. Then off spike continues to move fonward, the collision was occurred by plate teeth at high speed the second time, NO. 2 and No. 3 of the grain was removed from the broken down on the spike.

\subsection{Orthogonal Analysis of Grain Movement}

The movement track and the velocity were collected and processed by the MIDAS acquisition software and the EXCEL mapping tool, the movement track and velocity map of three grains were obtained. As shown in Figure 4 we can find the trajectory of the grain in Figure $4 \mathrm{a}$, from the motion curve and threshing cylinder axis direction angle of the three grain, we can judge from the hit efforts of grain and movement ability of 
tangentially after the strike. In specific, the angle between the axial direction and grain NO.1, NO.2, NO.3 were 80.02 degrees, 40.90 degrees and 45.58 degrees. Obviously, the angle of the NO.1 grain was the largest, which showed that the movement ability of the tangential direction of the NO.1 was better than that of the NO.2 and NO.3.

Figure $4 b$, Figure $4 c$ and Figure $3 d$ show the curve of the grain velocity for the before and after the off spike threshing, vt, vZ and $\mathrm{v}$ represent the tangent velocity, the speed of axial direction and the speed of synthesis, from the analysis of velocity, speed and direction for the three grain have been changed. Specifically, the speed for the NO.1 grain increase from $8.87 \mathrm{~m} / \mathrm{s}$ to $16.16 \mathrm{~m} / \mathrm{s}$ after the shock of high-speed rotation of the inner drum pads teeth, when the speed reaches to the $17.46 \mathrm{~m} / \mathrm{s}$, the movement area of grain is stable. At this time, the tangential velocity vt is about $20 \mathrm{~m} / \mathrm{s}$, the axial velocity is about $6 \mathrm{~m} / \mathrm{s}$, tangential velocity is more than 3 times of the axial velocity. Therefore, tangential velocity vt is the dominant factor of the velocity. Speed and movement direction of the NO.2 grain changes significant before and after the collision and the average speed is increased from $11.32 \mathrm{~m} / \mathrm{s}$ to $22.65 \mathrm{~m} / \mathrm{s}$ before collision. After the collision, tangentual velocity vt increase rapidly with increase of time, it is stable when it reaches about $16.78 \mathrm{~m} / \mathrm{s}$, variation range of the axial velocity $\mathrm{vz}$ is $8 \mathrm{~m} / \mathrm{s}-15 \mathrm{~m} / \mathrm{s}$ before and after the collision, variation amplitude is less than tangential velocity. Therefore, vt is dominant factors that constitute the speed; The Speed of the No. 3 grain changes 1 on $15.98 \mathrm{~m} / \mathrm{s}$ to $120.87 \mathrm{~m} / \mathrm{s}$ before and after the collision, the range of movement velocity of No. 3 grain is the largest, the smooth movement is better than threshing.
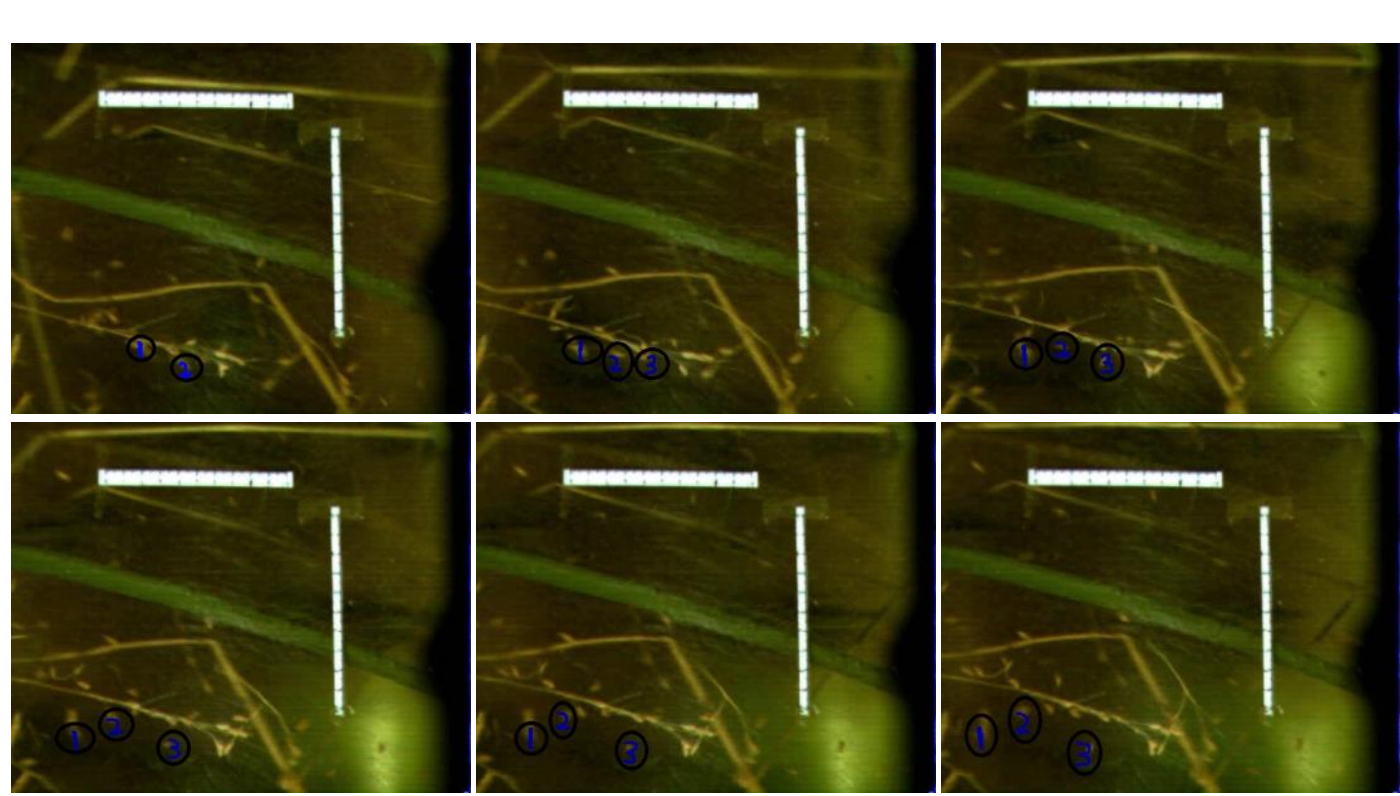

Figure 3. Broken Threshing Process of Spike 


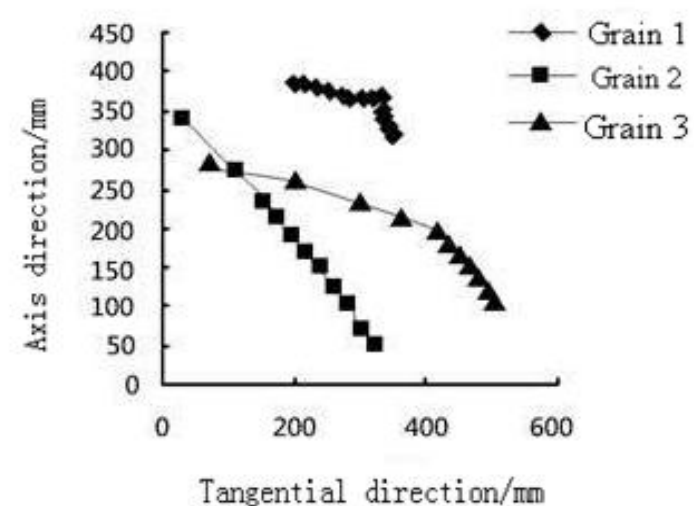

(a)

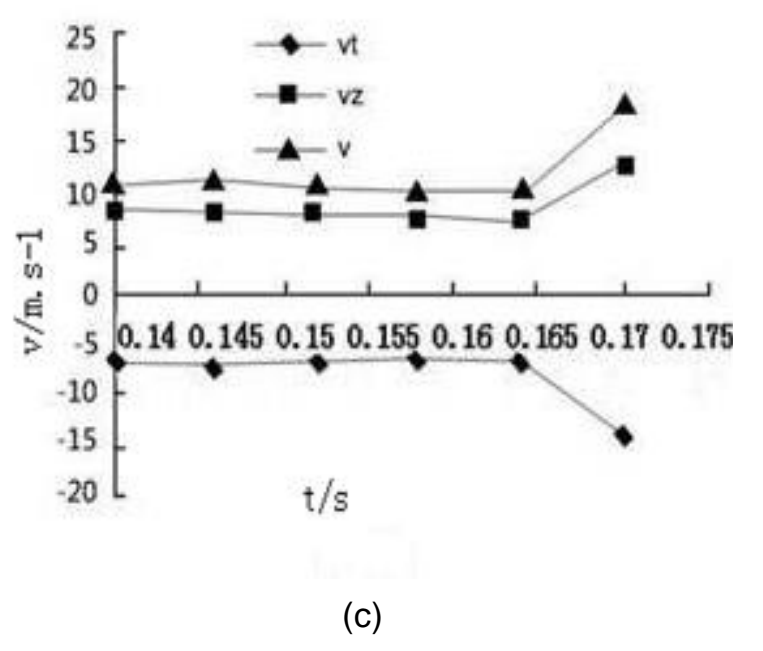

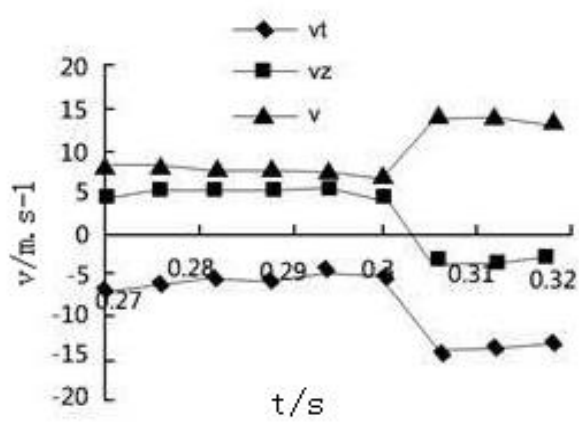

(b)

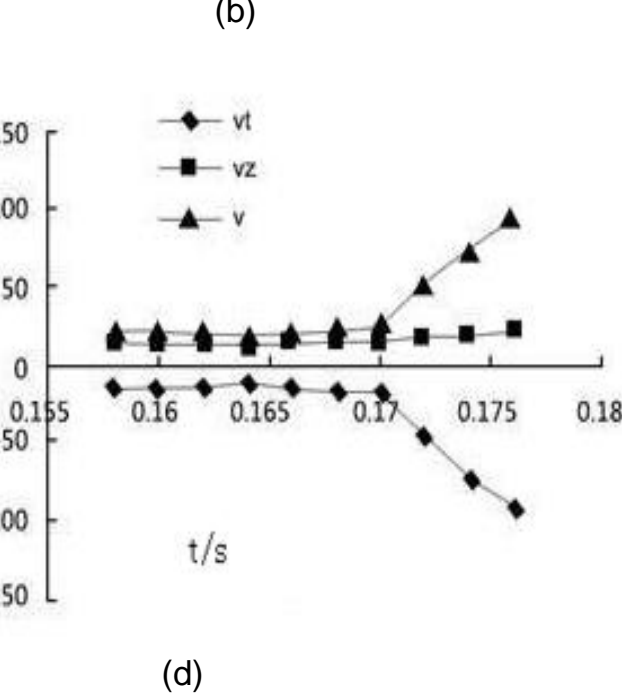

Figure 4. Motion Trace and Velocity of Kernels Threshed from Broken Ear

\subsection{Analysis of Free Grain Movement under Different Rotating Speed}

When the feeding amount was $0.5 \mathrm{~kg} / \mathrm{s}$, the rotation speed of the roller was $500 \mathrm{r} / \mathrm{min}$ and $600 \mathrm{r} / \mathrm{min}$, respectively, the ten free grains were collected in the rice sub space, and the track and the velocity were shown in Figure 5 and Figure 6. From the movement trajectory of free grain in Figure 5a and Figure 6a, firstly, in the absence of outside interference, the majority of the motion trajectory of free grains in plate tooth axial off space is a straight line, and a certain angle with the axial direction of the continuous forward movement along the axial direction of the drum, the angle varies quite different. Secondly, the majority of free grains were forward movement in the free space, and the movement direction of a few free grains was reverse.

From Figure $5 \mathrm{~b}$ and Figure $6 \mathrm{~b}$, the motion state of the free grain for three roller speed in stripped space can be divided into three forms: high speed, medium movement and low movement. The most of free grain in the removal of the sub space is in medium speed motion. When the rotational speed of the roller has been changed, the movement speed of free grain also changes. When the roller speed increases from the $500 \mathrm{r} / \mathrm{min}$ to $600 \mathrm{r} / \mathrm{min}$, the speed of free grain will change from $10-20 \mathrm{~m} / \mathrm{s}$ to $12-30 \mathrm{~m} / \mathrm{s}$. Therefore, synthesis speed of the free grain increases with the increase of the drum speed. 

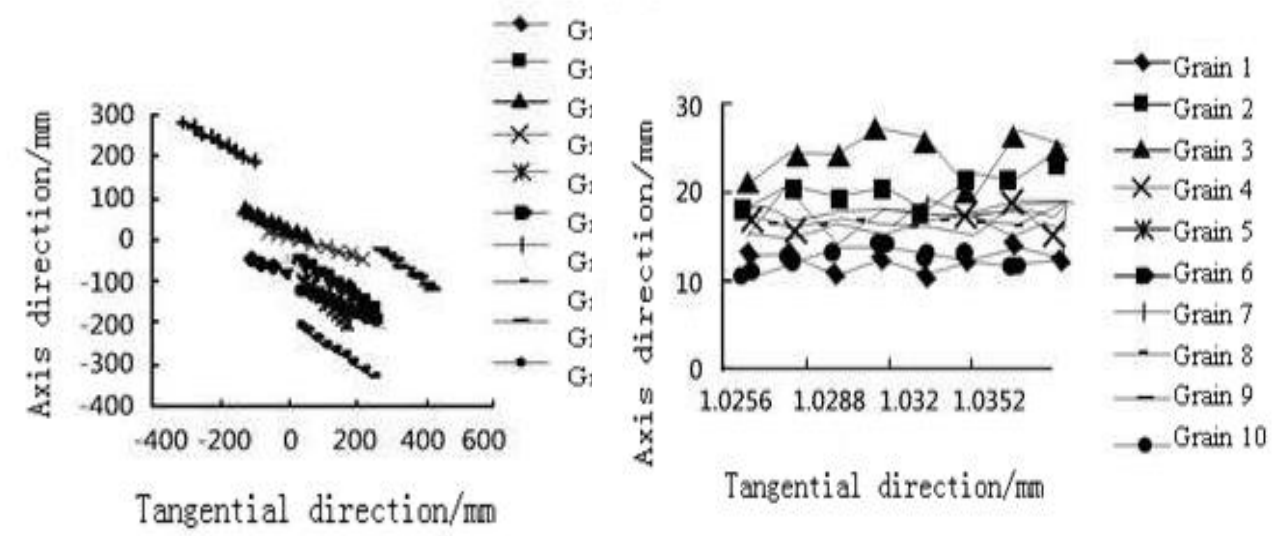

(a)

Figure 5. Grain Trajectory and Velocity Speed 500r/Min

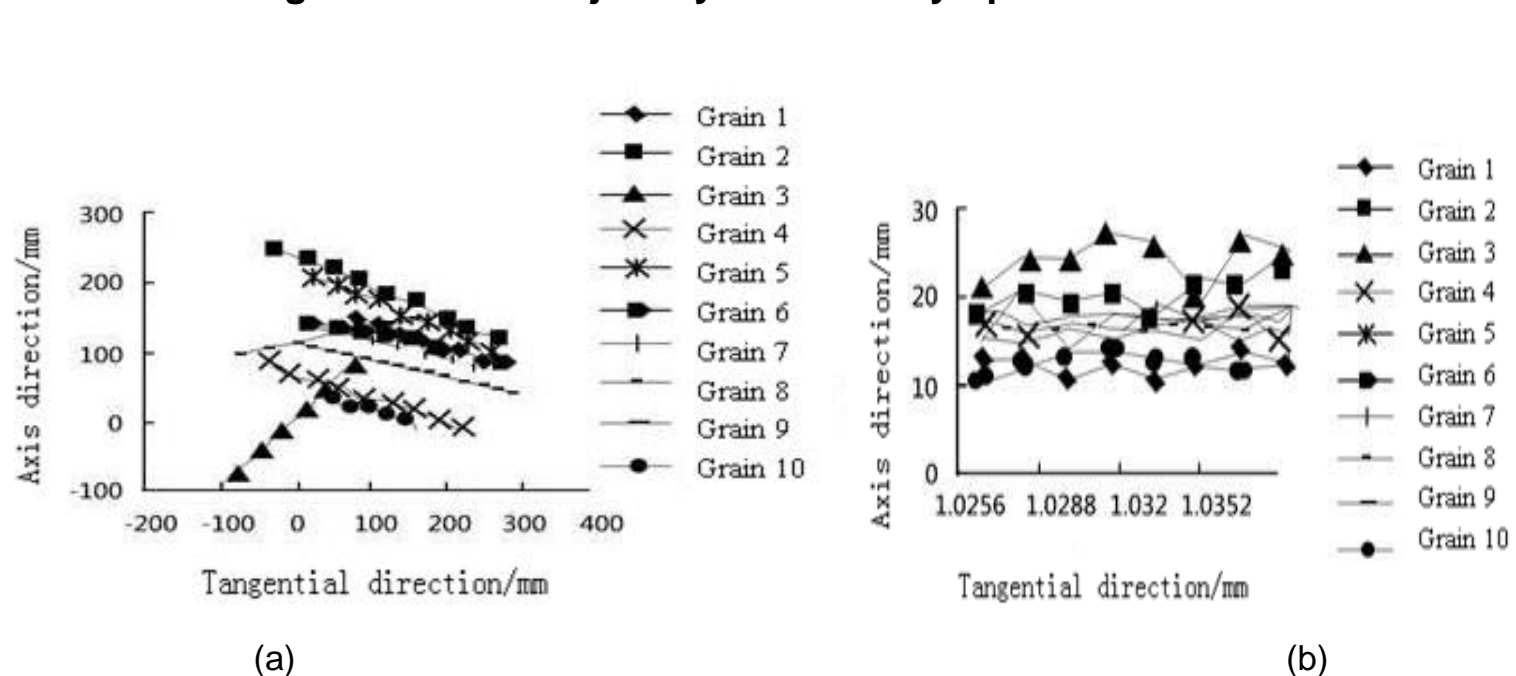

(a)

Figure 6. Grain Trajectory and Velocity Speed 600r/Min

\section{Distribution Rules of Rotors at the Axis Direction}

\subsection{Test Conditions}

Under inftial conditions: concave clearancea $30 \mathrm{~mm}$, and cylinder speed $600 \mathrm{r} / \mathrm{min}$, zenith distance $30 \mathrm{~mm}$, grain moisture content $15 \%$, and feeding amount $2 \mathrm{~kg} / \mathrm{s}$, distribution rules at the axis direction was tested.

\subsection{Analyzing the Results}

Figure 7 is distribution curve of rotors at the axis direction. Comparing Figure 7 with Figure 2, which is stress distribution at the axis direction, it is concluded that two conclusions: (1) both fluctuates at the axis direction and distribution is uneven; (2) stress appears two peak values at the axis direction, respectively in early stage of the threshing and at the distance of about 1.3 meters from the feeding end. An area at the distance of about 1 1.7 meters from the feeding end along the axis direction is a rotors peak area, where duration of stress peak value is longer, and with certain fluctuations, and rotors distribution at the axis direction first increased and then reduced. 
Based on the above analysis results, we can see that grains enter the device, under the action of kneading and extrusion of threshing parts such as spiral vane to seperate the grain and stem, and rotors constantly are expelled from the concave board into material pick-up vehicle. In grain threshing and separating process, seperating process of rotors through seperating device such as the concave board is one of important reasons why results in difference of distribution rules of stress and rotors at the axis directon. The seperating process includes three steps: (1) a part is separated instantly; (2) the other part of grains and stems enter the next stage to thresh; (3) the rest with the straws are discharged.

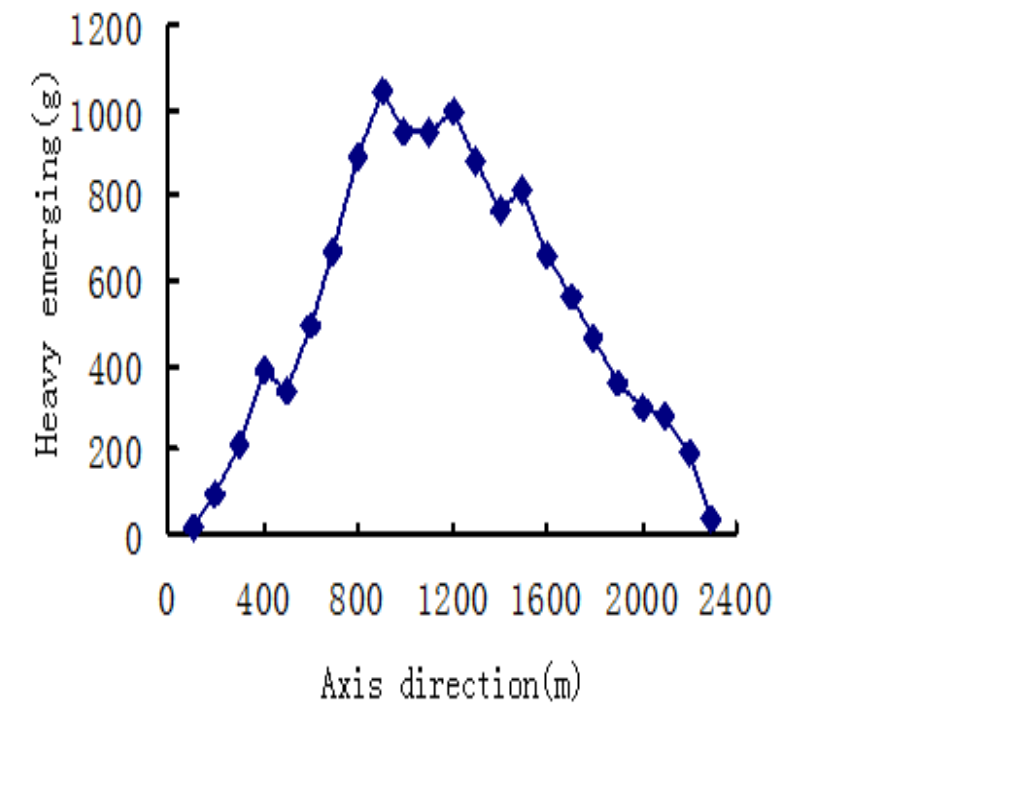

Figure 7. Distribution Curves of Mixture along Axial Directions

\section{Conclusion}

Stress model was set based on the stress principle in this paper, with structure characteristies of assembled axial flow threshing and separating device, through simulating analysis in Matab, we can obtain the trend of fluctuations in grain threshing and separating process and the characteristics that change is uneven. At the same time, two peak values appear at early and middle threshing stage respectively; threshing and separating proces finishes about 2.35 meters from the feeding mouth at axial direction. Test study valifies that rotors distribute along the axis direction, and the test results are consistent with the theory. At the same time, it is concluded from grain movement analysis that, in the absence of interference from the outside conditions, the free grain in the free grain movement is basically linear movement. Under the certain conditions, most free grain in the threshing and separating space is in a straight line motion approximation, Which is in the medium speed motion state. The track, velocity and direction of movement have been changed after the collision between grain and plate. At the same time, the movement speed of the grain is related to the speed of the roller, movement speed increase while the roller's speed increases.

In this paper, study reveals that the stress distribution rules and the nature of threshing and separating, and provides the theoretical basis for choosing the most suitable components and optimizing system. 


\section{Acknowledgments}

Project Support:

1. State "twelfth five-year" science and technology support project (2014BAD06B01-09) Rice de-seeding device key technology research ;

2. State "twelfth five-year" science and technology support project (2014BAD06B01-06) Large-scale rice segmented harvest key technology and equipment

3. State "twelfth five-year" science and technology support project (2014BAD06B01-08) Rice windrower research key technology and parameters;

\section{References}

[1] G. Tao and S. Yi, "Distribution Rules of Grain Rotors with Combined Axial Flow Device", Journal of agricultural mechanization research, Los Angeles, vol. 4, (2009), pp. 134- 136.

[2] V. M. Huynh and T. Powell, "Threshing and Particulary in Process-a Mathematical Model", The Transactions of the ASAE. J, Los Angeles, vol. 20, (1982), pp. 65 -73.

[3] G. F. Kamst, C. Bonazzi and J. Vasseur, "The Effect of Deformation Rate and Moisture Content on the Mechanical Properties of Rice grains", Trans of the ASAE, Los Angeles, vol. 1, (2002) pp 145-151.

[4] S. Yi and E. Jiang, "High-speed Video Analysis in Threshing Prootess of Axial Flow Threshing and Separating Equiptment", Journal of agricultural machinery, Los Angeles, vol. 5, (2008), pp.35-38.

[5] S. Yi, G. Tao and X. Mao, "Simulation Study on Dynamic Model of Combined Axial Flow Device", Journal of agricultural engineering, Los Angeles, vol. 7, (2009), pp.38-41.

[6] G. Tao. "Simulation and Experimental Research of Combined Axial Flow Device", Daqing, Heilongjiang Bayi Argriculture University, Los Angeles, (2008).

[7] X. Mao, "Grain Flow Motion Model and Simulation Study of Combined Axial Flow Device", Daqing, Heilongjiang Bayi Argriculture University, Los Angeles, (2008).

\section{Liu Ying-Nan}

Author's profile: LIU Ying-nan (1982-SHeilongjiang Province, Anda. Lecturer. Master.

Email:dqpilyn@126.com.Telphone:15845962618

Correspondent author. YI Shu-juan (1965-), Shandong Province, Qixia. Professor. doctoral supervisor.

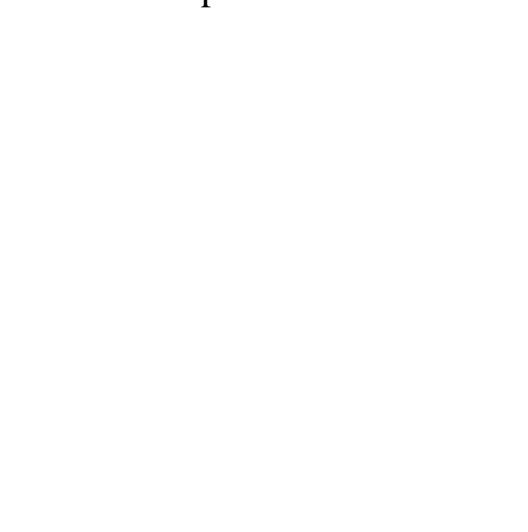

